\title{
Tactile selective attention and body posture: Assessing the multisensory contributions of vision and proprioception
}

\author{
SALVADOR SOTO-FARACO \\ University of Oxford, Oxford, England \\ and Universitat de Barcelona, Barcelona, Spain \\ ANGELICA RONALD \\ University of Oxford, Oxford, England \\ and Institute of Psychiatry, London, England \\ and \\ CHARLES SPENCE \\ University of Oxford, Oxford, England
}

\begin{abstract}
This study addressed the role of proprioceptive and visual cues to body posture during the deployment of tactile spatial attention. Participants made speeded elevation judgments (up vs. down) to vibrotactile targets presented to the finger or thumb of either hand, while attempting to ignore vibrotactile distractors presented to the opposite hand. The first two experiments established the validity of this paradigm and showed that congruency effects were stronger when the target hand was uncertain (Experiment 1) than when it was certain (Experiment 2). Varying the orientation of the hands revealed that these congruency effects were determined by the position of the target and distractor in external space, and not by the particular skin sites stimulated (Experiment 3). Congruency effects increased as the hands were brought closer together in the dark (Experiment 4), demonstrating the role of proprioceptive input in modulating tactile selective attention. This spatial modulation was also demonstrated when a mirror was used to alter the visually perceived separation between the hands (Experiment 5). These results suggest that tactile, spatially selective attention can operate according to an abstract spatial frame of reference, which is significantly modulated by multisensory contributions from both proprioception and vision.
\end{abstract}

How the different sensory modalities are coordinated for the efficient control of perception and action is a key question in selective attention research (e.g., Driver \& Spence, 1998a). This is a nontrivial problem, given that sensory information is initially encoded according to the intrinsic characteristics of each receptor system (i.e., visual information is initially encoded retinotopically and tactile information is encoded somatotopically, whereas auditory information is encoded tonotopically). Information from different sensory modalities presumably needs to be remapped in a common frame of reference in order to allow for the coordination of multisensory inputs and the effective deployment of attention in space (see Spence \& Driver, 2004). The present study addressed the question of how tactile spatial information is

This study was supported by a Network Grant from the McDonnellPew Centre for Cognitive Neuroscience in Oxford to S.S.-F. and C.S. Correspondence regarding this article should be addressed to either S. Soto-Faraco, Department de Psicologia Bàsica, Universitat de Barcelona, Pg. de la Vall d'Hebrón, 171, 08035 Barcelona, Spain (e-mail: ssoto@psico.psi.ub.es) or to C. Spence, Department of Experimental Psychology, South Parks Road, Oxford OX1 3UD, England (e-mail: charles.spence@psy.ox.ac.uk). remapped as a function of proprioceptive and visual information during a spatial selective attention task.

Numerous studies have shown that a person's ability to detect or identify a tactile stimulus presented to a particular location on the skin can be impaired if a distractor stimulus is presented from a nearby skin site (e.g., Craig, 1974; Gilson, 1969; Horner, 1995, 1997; Weisenberger, 1994; Weisenberger \& Craig, 1982). Such tactile interference effects do not merely reflect the consequences of sensory masking, since an attentional component to the effect has also been identified (e.g., Craig, 1974; Evans \& Craig, 1991; Horner, 1997, 2000). Moreover, in accord with previous studies of distractor interference effects in other sensory modalities (such as vision or audition; see, e.g., Chan, Merrifield, \& Spence, 2004; Eriksen \& Eriksen, 1974; see Styles, 1997, for a review), somatosensory interference effects have been shown to decline as the spatial distance between the target and the distractor/mask is increased. However, the sense of touch presents a particularly intriguing case for research on selective attention, because the distance between a target and a distractor can be defined according to more than one frame of reference. Given that tactile receptors on the hand can be 
moved to a variety of different external locations with respect to the body, trunk, and head, a tactile stimulus presented to a particular skin site (i.e., the hand) can potentially come from a wide range of different locations in external space. Therefore, one important issue that arises regards which frame(s) of reference are relevant for determining the spatial distribution of tactile selective attention (see Kim \& Cruse, 2001; Lakatos \& Shepard, 1997).

One possibility is that tactile spatial attention operates at a stage of information processing at which the stimuli are represented according to a somatotopic frame of reference and that the distance between the target and the distractor would, therefore, reflect the separation between two points across the body/skin surface (regardless of the posture adopted). This would seem a likely possibility, given that this frame of reference corresponds to the coordinate system in which tactile stimuli are initially organized in the somatosensory cortex (e.g., Kim \& Cruse, 2001; Penfield \& Rasmussen, 1950). Alternatively, tactile attention may operate within a more abstract coordinate system (i.e., without a direct dependence on the particular skin sites being stimulated), in which the distance between the stimuli is defined in terms of their separation according to a frame of reference centered in the head or the trunk (egocentric frame of reference) or in terms of their relative position in external space (allocentric frame of reference). The spatial coding of tactile stimuli in a more abstract frame of reference (i.e., egocentric or allocentric, rather than somatotopic) would presumably be very useful during our interaction with the environment (i.e., when engaged in such behaviors as haptic exploration). However, in order for tactile attention to operate within such an abstract coordinate system, information regarding the skin site stimulated (encoded in somatotopic coordinates) must be integrated with cues about body posture provided by the other senses (such as proprioception, vision, and possibly also audition). Our goal in the present study was to investigate the nature of the frame of reference used during tactile selective attention.

\section{Evidence for a Somatotopic Frame of Reference}

The results of a study of tactile inhibition of return (IOR) reported by Röder, Spence, and Rösler (2000) suggest an important role for a somatotopic frame of reference in tactile reflexive attentional orienting. IOR is the name given to the slowing of detection responses to a target stimulus when it is presented at, or near to, a previously cued location, once any facilitatory effects of the cue (attributable to an exogenous shift of attention toward the cued location) have dissipated (typically after a few hundred milliseconds of the onset of the cue; see, e.g., Klein, 2000; Posner \& Cohen, 1984; see Spence, Lloyd, McGlone, Nicholls, \& Driver, 2000, for a demonstration of IOR between all combinations of auditory, visual, and tactile cue and target stimuli). In Röder et al.'s study, the tactile cue and target stimuli were presented randomly to the fingers of either hand. Reaction times (RTs) were slowest (i.e., the IOR effect was strongest) when the cue and the target were presented to the same digit, whereas response latencies were also slowed (albeit to a lesser extent) when the cue and the target stimuli were presented from adjacent digits on the same hand. Crucially, however, there was no evidence of IOR when the cue and the target were presented to different hands, even if the stimulated digits on the two hands were placed directly next to each other (i.e., at the same physical distance in external space at which IOR had been found following stimulation of adjacent fingers on the same hand). These results suggest that the spatial distribution of tactile IOR can be constrained by the distance in somatotopic space.

In another influential study, Evans and Craig (1991) evaluated tactile endogenous (i.e., voluntary) selective attention as a function of somatotopic distance. The participants in their study were required to discriminate the direction of motion (left vs. right) of a pattern presented to one fingertip while attempting to ignore irrelevant motion information (congruent or incongruent with the target motion) delivered to another fingertip. The interference between target and (conflicting) distractor motion on the incongruent trials was weaker when the distractor was presented to a finger on the nontarget hand than when it was presented to a different digit on the target hand. In fact, no significant distractor interference was found when the target and the distractor were delivered to different hands. Since the distance between the target and the distractor fingertips in Evans and Craig's study was kept constant in terms of external space, their results support the idea that the spatial distribution of tactile attention may be determined by somatotopic coordinates.

In a subsequent study, Evans, Craig, and Rinker (1992) used the same motion discrimination task to measure selective tactile attention between adjacent and nonadjacent fingers of the same and opposite hands. They reported similar interference effects whether the target and the distractor stimuli were presented to adjacent or nonadjacent fingers of the same hand, suggesting a relatively broad tuning of the attentional focus to the hand (i.e., perhaps reflecting a hand-centered attention effect). Just as in Evans and Craig's (1991) study, interference effects dropped dramatically when the target and the distractor stimuli were presented to different hands, although they were still present in that condition (contrasting with the results of Evans and Craig's study). Interestingly, however, when the targets and the distractors were presented to different hands, Evans et al. found no difference between the magnitude of interference effects reported when the two hands were placed close together (separated by $10 \mathrm{~cm}$ ) versus when they were placed farther apart (separated by $42 \mathrm{~cm}$ ). This null effect of hand separation again supports the view that the spatial modulation of tactile selective attention occurs within a somatotopic, rather than within an externally defined (or allocentric), frame of reference. 


\section{Evidence for an Abstract Frame of Reference}

However, not all previous studies have reported results that are consistent with a purely somatotopic basis to tactile selective attention. In fact, the results of several studies suggest that the coordination of tactile spatial attention may also be determined by a more abstract (be it egocentrically or externally based) frame of reference, at least under certain circumstances (e.g., Aglioti, Smania, \& Peru, 1999; Driver \& Grossenbacher, 1996; Lakatos \& Shepard, 1997; Moscovitch \& Behrmann, 1994; Rinker \& Craig, 1994; Tinazzi, Ferrari, Zampini, \& Aglioti, 2000). For example, Moscovitch and Behrmann (see also Tinazzi et al., 2000) studied a group of neurological patients suffering from tactile extinction (a syndrome usually associated with damage to the right parietal cortex, resulting in an attentional deficit in responding to stimuli presented on the left side of space). In their study, patients were stimulated on the ipsilesional (right) and contralesional (left) sides of their ipsilesional (right) wrist while assuming one of two postures: either with the palm facing downward or with the palm facing upward. Moscovitch and Behrmann found that patients frequently missed tactile stimuli on the left side of their wrist (according to external coordinates), regardless of the hand posture adopted. That is, neglect occurred or ceased to occur at a particular skin site, depending on the different hand orientations adopted. This suggests that the attentional deficit underlying extinction in these patients affected stimuli presented on the side of space contralateral to the lesion.

Rinker and Craig (1994) reported a series of experiments conducted on healthy normal participants whose results point to the same conclusion. The participants in their study had to discriminate the direction of motion of a tactile stimulus that moved across the thumb, while trying to ignore the direction of a stimulus moving across the index finger. The direction of distractor motion could be either congruent or incongruent with that of the target. Rinker and Craig tested participants as they adopted one of two different postures: either placing their hand palm down flat on the table (i.e., with the digits resting on top of the tactile arrays), or else holding a cube in their hand (with the finger and thumb resting on the tactile arrays placed flat on opposite sides of the cube). The relative congruency of the motion direction in external coordinates changed as a function of the change of posture (i.e., what was congruent in one posture was incongruent in the other posture, and vice versa), but not in terms of somatotopic coordinates. ${ }^{1}$ Rinker and Craig found that congruency effects were determined by the direction of motion of the stimuli in external spatial coordinates (rather than by the direction of motion across the digits, which should have been unaffected by the change of posture).

Taken together, the results of the research reviewed so far are consistent with the view that tactile spatial attentional effects are determined by different frames of reference in different situations. In particular, it appears that an abstract frame of reference may be prevalent when combinations of stimuli are presented to a particular hand and all that varies is the relative orientation of the hand in space (e.g., Moscovitch \& Behrmann, 1994; Rinker \& Craig, 1994; Tinazzi et al., 2000). However, somatotopic coordinates (or even hand-centered attentional effects) determine performance under conditions in which the target and the distractor stimuli are presented to different hands (i.e., in bimanual stimulation conditions) and what varies is the relative position of the two hands in space (e.g., Evans \& Craig, 1991; Evans et al., 1992; Röder et al., 2000).

However, there are other results that suggest an abstract representation of tactile space during attentional selection even when it is the separation between the hands that is manipulated. For example, Lakatos and Shepard (1997) found that the time required for participants to shift their attention from monitoring stimuli presented to one wrist to detecting an unexpected target presented to the opposite wrist was longer when they held their two arms fully outstretched to the sides of their trunk (i.e., adopting the posture that maximized the separation between the hands) than when they held their arms extended forward (i.e., the hands were held in close proximity to each other). These results suggest that one of the main determinants of the amount of time required to switch attention between different body sites is the $e x$ ternal distance between the two points, rather than just the somatotopic distance traversed across body surface (which was constant across the two postures tested). At first sight, these data seem to conflict with Evans et al.'s (1992) results suggesting that it is only the distance in a somatosensory frame of reference that modulates the distribution of tactile, spatially selective attention. However, there are important methodological differences between these two studies, including the paradigm used (focused attention vs. attention switching) and the distance between hands that was tested in the far condition of each study (which was considerably smaller in Evans et al.'s study than in Lakatos and Shepard's study). For this reason, direct comparison of these experiments is difficult.

Driver and Grossenbacher (1996) reported another study that also highlighted a significant role of distance in terms of an abstract frame of reference. They used a selective attention task, and therefore, their paradigm was more similar to that used by Evans et al. (1992) than to that used by Lakatos and Shepard (1997). The participants in their study had to discriminate a target (single vs. double vibration) presented to the little finger of one hand, while ignoring a concurrent vibration (congruent or incongruent with respect to the target) presented to the little finger of the opposite hand. Driver and Grossenbacher found that selective attention to a hand was not perfectly efficient, since they observed significant effects of congruency from the distractor presented to the opposite hand, thus confirming Evans et al.'s finding regarding the existence of tactile interference across the hands. A second, and more important, finding to emerge from 
Driver and Grossenbacher's studies was that the magnitude of the congruency effect decreased when the participants placed their hands far apart. Since the distance in terms of somatotopic space did not change, their result suggests, again, that distance in egocentrically or externally determined coordinates modulated the effects of tactile selective attention. This result, however, stands in contrast with Evans et al.'s findings. The reasons for such a discrepancy are most likely based on methodological differences between the two studies, perhaps among the most relevant being the larger difference in hand separation between the hands-near and the hands-far conditions used by Driver and Grossenbacher $(66 \mathrm{~cm}$ in their study, as opposed to $32 \mathrm{~cm}$ in Evans et al.'s study).

\section{Scope of the Present Study}

The aim of the present study was to address several questions that remain currently unresolved with regard to the frame of reference in which tactile spatial attention operates. Our first goal (in Experiments 1 and 2) was to test spatial selective attention in touch under conditions of unpredictable versus predictable target location. In the majority of previous studies (e.g., Driver \& Grossenbacher, 1996; Evans \& Craig, 1991; Evans et al., 1992; Rinker \& Craig, 1994), target location was known to the participants in advance, and therefore, tactile selective attention may have been more effective. However, in most real-life situations involving haptic exploration, people use several fingers at the same time to search a surface for a given feature, without necessarily having any advance knowledge of which finger will first encounter the target. Moreover, one might think that the interference from irrelevant distractors would be larger under conditions of spatial uncertainty than under conditions of spatial certainty typically used in other previous research. The second goal of the present study was to examine tactile selective attention in space as a function of body position and to assess the contribution of proprioceptive cues to the remapping of tactile space. We approached the influence of posture on tactile spatial attention with two complementary manipulations. In Experiment 3 , we introduced a postural manipulation that led to a change in the somatotopic frame of reference while leaving the location of the stimuli fixed in terms of external and egocentric coordinates across conditions. In Experiment 4, we tested the reverse situation: The external location of the stimuli changed while their position in terms of somatotopic coordinates remained fixed. Finally, in Experiment 5, we addressed the potential influence of visual cues to body posture while maintaining the stimuli at a fixed location in terms of somatotopic, egocentric, and external space.

In the present study, we used an elevation discrimination task. The task was specifically chosen because it has proven to be remarkably sensitive to both intramodal and cross-modal cuing effects in previous attentional studies (e.g., Kennett, Eimer, Spence, \& Driver, 2001; Kennett, Spence, \& Driver, 2002; Spence \& McGlone, 2001;
Spence, Pavani, \& Driver, 2000), as well as providing a robust measure of distractor interference in cross-modal studies (Spence, Pavani, \& Driver, 1998; see Spence, Pavani, Maravita, \& Holmes, 2004, for a recent review). We started by testing the adequacy of this task for studying spatial selective attention in touch.

\section{EXPERIMENT 1}

Given the conflicting findings in previous research regarding people's ability to ignore tactile distractors presented to one hand while trying to respond to tactile targets presented to the other hand (e.g., Driver \& Grossenbacher, 1996; Evans \& Craig, 1991; Evans et al., 1992), our first experiment was conducted in order to determine whether participants would indeed be any slower (and/or less accurate) in making elevation discrimination responses to vibrotactile targets presented to one hand when conflicting vibrotactile distractors were presented to the opposite hand.

Participants held a foam cube in each hand between the index finger and the thumb. On each trial, a continuous vibrotactile target was presented to the index finger or the thumb of one hand and a pulsed vibrotactile distractor to the index finger or the thumb of the other hand. The participant was required to discriminate the elevation (up vs. down, respectively) of the vibrotactile targets as rapidly, and accurately, as possible. Any failure of tactile selective attention (i.e., in terms of an inability to ignore the elevation of the distractor vibrations) should reveal itself in terms of slower and/or less accurate responding on trials in which the target and the distractor were presented from incongruent elevations (i.e., an upper target to the index finger of one hand and a lower distractor to the thumb of the other hand), as opposed to being presented from congruent elevations (i.e., when both the target and the distractor were presented to corresponding digits on different hands).

In this experiment, however, we introduced a novelty with respect to the previous tactile selective attention studies cited in the introduction. In order to maximize the likelihood of finding a congruency effect, we presented the target and the distractor stimuli unpredictably to either hand, instead of presenting them from fixed locations (i.e., on a particular hand; see, Horner, 1997; Meyer, Gross, \& Teuber, 1963). In the context of this experiment, we reasoned that by making the target location unpredictable, the participants would have to divide their attention between both hands (see Horner, 1997, for a similar argument regarding different locations within the same finger). Hence, they should find it harder to ignore the irrelevant distractor than if they had been certain of the target hand in advance and, so, had been able to focus their attention selectively on just one (target) hand.

\section{Method}

Participants. Eight psychology students ( 7 men and 1 woman) from the University of Oxford were recruited to take part in the experiment. All were naive as to the purpose of the experiment. Their 
mean age was 25 years (range, 20-32 years), and all were righthanded by self-report. All reported normal tactile sensitivity and normal or corrected-to-normal vision.

Apparatus and Materials. The participant sat at a table in a dark and quiet room, holding two foam cubes placed $30 \mathrm{~cm}$ apart and equidistant from the midline. Two vibrotactile stimulators (Oticon-A bone conduction vibrators; Hamilton, U.K.) were embedded into the front top and bottom edges of each cube (see Figure 1). The participant placed his or her index finger over the upper vibrator and the thumb over the lower vibrator on each cube. Vibrotactile targets consisted of a clearly suprathreshold $200-\mathrm{Hz}$ sine wave signal presented continuously for $300 \mathrm{msec}$ from one of the four vibrators, while the vibrotactile distractors consisted of the pulsed presentation of a $200-\mathrm{Hz}$ sine wave signal (three 50-msec bursts, each separated by $50-\mathrm{msec}$ off periods) at the same intensity. White noise was presented to the participant over headphones throughout each block of experimental trials at an intensity sufficient to mask any noise produced by the operation of the vibrotactile stimulators.

A red light-emitting diode (LED) served as a central fixation point placed $48 \mathrm{~cm}$ in front of the participant's eyes, at an angle of approximately $33^{\circ}$ below eye level. Maintaining gaze direction constant across conditions and throughout the experiment is important, since some studies have shown that gaze direction can have important modulatory effects on tactile attention (e.g., Driver \& Grossenbacher, 1996). Four LEDs, one placed next to each vibrotactile stimulator, were used to present visual feedback following an erroneous response. The participant made speeded elevation discrimination responses by means of two foot pedals placed under the table, one below the toes, and the other below the heel, of the right foot. A custom software program was used to control the presentation of stimuli and register the participant's responses by means of a specialized PC interface.

Procedure. The participant was instructed to maintain fixation on the central LED throughout each block of trials. He or she was instructed to discriminate the elevation of the continuous vibrotactile targets (upper vs. lower) as rapidly and accurately as possible, while trying to ignore the pulsed distractor vibrations presented si- multaneously to the other hand. The participant responded to upper targets by briefly depressing the response pedal under the toe of the right foot and to lower targets by lifting the foot off the other pedal placed under the right heel. The four feedback LEDs were flashed briefly (eight 100-msec flashes, each separated by 20 -msec off periods) whenever the participant made an incorrect response. If a response had not been initiated within $6 \mathrm{sec}$ of target onset, the feedback LEDs were illuminated continuously for $960 \mathrm{msec}$. A new trial was initiated after a further delay of 900-1,050 msec.

The experimental session was initiated with three blocks of 20 practice trials (which were not analyzed), followed by three experimental blocks of 64 trials. In the first practice block, the participant had to judge the elevation of isolated vibrotactile targets presented randomly to either hand (i.e., no distractors were presented). In the second practice block, the targets were always presented to the same hand (with the side being counterbalanced across participants) and the distractors always being presented to the other hand. The third practice block was equivalent to the experimental blocks of trials, in which the target on each trial was presented unpredictably to either hand, while the distractor was always presented to the other hand. An equal number of vibrotactile target and distractor stimuli were presented to each of the four possible stimulus locations in each block of trials. Congruent and incongruent trials were equiprobable, and their order of presentation was randomized for every participant.

\section{Results and Discussion}

We assessed the mean correct RT and accuracy for every participant and condition (see Figure 2A for group averages in each condition). In this and all the subsequent analyses reported in this article, we eliminated trials on which the RT fell outside $\pm 2 S D$ s from the average RT of a particular participant in a particular condition (this led to the removal of $5 \%$ of the RT data in this experiment). A one-way analysis of variance (ANOVA) on the

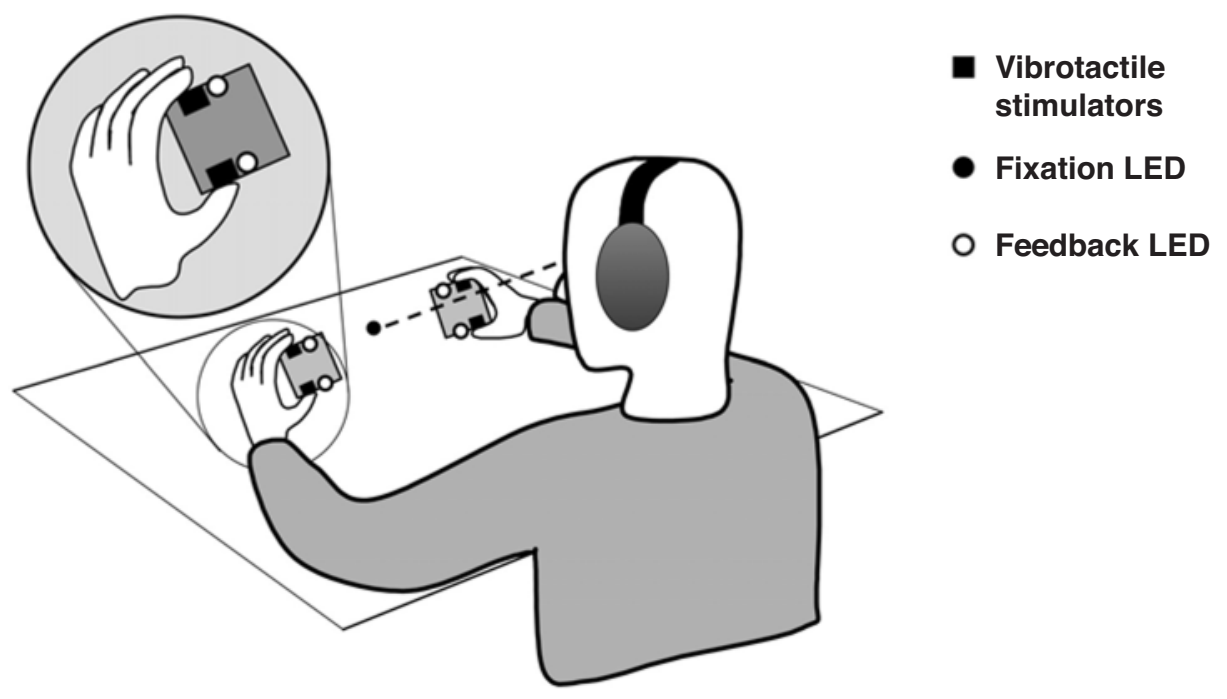

Figure 1. Schematic representation of the experimental layout used in Experiment 1. The participant held two foam cubes, each incorporating two vibrotactile stimulators, one contacting the index finger and the other contacting the thumb (see inset to the left). LEDs placed next to each vibrotactile stimulator provided feedback following erroneous responses. The participant made speeded responses by momentarily lifting the right foot off one of the two pedals under the toe and the heel of the right foot (not shown). White noise was presented constantly to the participant though headphones, in order to mask any sounds made by the operation of the vibrotactile stimulators. 


\section{(A) Experiment 1 (unpredictable target location) \\ (B) Experiment 2 (predictable target location)}
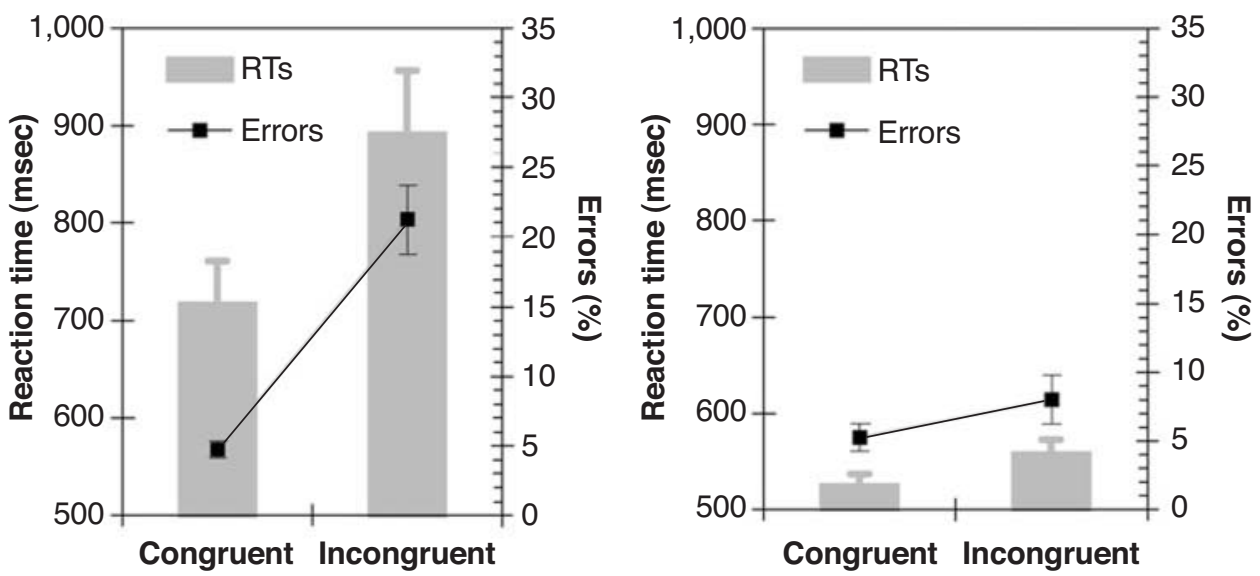

Figure 2. The results of the vibrotactile elevation discrimination task as a function of target-distractor congruency for (A) Experiment 1 (unpredictable target hand) and (B) Experiment 2 (predictable target hand). The bars represent the mean reaction times (RTs; see the left-hand side axis), whereas the filled squares connected by solid lines represent the percentages of errors (see the right-hand side axis). The error bars represent the standard errors of the means.

mean RT data, including the within-subjects factor of distractor congruency (congruent vs. incongruent), revealed that responses to congruent trials were significantly faster than those to incongruent trials [mean congruency effect of $173 \mathrm{msec} ; F(1,7)=12.6, p=.009$ ]. The analysis of the error data revealed that the participants were also significantly more accurate on congruent trials than on incongruent trials overall [mean congruency effect of $16 \% ; F(1,7)=19.5, p=.003$ ].

The results of Experiment 1 therefore demonstrated a failure of tactile, spatially selective attention in a bimanual distractor task. That is, the participants were unable to ignore the elevation of the pulsed vibrotactile distractor stimuli presented to one hand when trying to respond to the elevation of the continuous vibrotactile targets presented to the opposite hand. The robust tactile congruency, or interference, effect present in both the RT and the error data replicated similar interference effects reported in several previous studies (e.g., Driver \& Grossenbacher, 1996; Evans et al., 1992; but see Evans \& Craig, 1991), but here using a novel elevation discrimination task. The results of Experiment 1 also suggested that the elevation discrimination task would be suitable for use in our subsequent experiments in which the possible modulatory role of proprioceptive and visual inputs in this putatively intramodal tactile, spatially selective attention task was examined. However, because most previous studies had used predictable target locations, it seemed worthwhile to determine what influence target location uncertainty might have on tactile elevation discrimination performance in this task before moving on to look at any such multisensory contributions to performance.

\section{EXPERIMENT 2}

Target location (i.e., hand) was made uncertain in our first experiment in order to try and maximize any congruency effects reported. At first sight, it would appear that the effects reported in Experiment 1 (unpredictable target location) are larger than comparable manipulations reported in certain previous studies (e.g., Evans \& Craig, 1991; Evans et al., 1992; but see also Driver \& Grossenbacher, 1996). However, direct comparison with these earlier studies is difficult, given the numerous methodological differences between them. We decided to assess what contribution, if any, spatial uncertainty had in determining the overall magnitude of tactile congruency effects, using the same method as that in Experiment 1 . Therefore, the participants once again performed the elevation discrimination task, but now (and in contrast to Experiment 1) the target and distractor hands were fixed for each block of trials. The participants were informed of this fact and were given clear instructions prior to the start of each block of trials as to which side the target and distractor stimuli would be presented from. Our prediction was that the magnitude of any congruency effects would be reduced in this second experiment under conditions of advance knowledge regarding target and distractor location.

\section{Method}

Participants. Eight psychology students from the University of Oxford ( 2 men and 6 women) took part in this experiment. Their mean age was 21 years, ranging from 19 to 23 years. Six of the participants were right-handed by self-report. The participants were naive as to the purpose of the experiment, and none had taken part 
in Experiment 1. All the participants reported normal tactile sensitivity and normal or corrected-to-normal vision.

Apparatus, Materials, Design, and Procedure. The apparatus, materials, design, and procedure were exactly the same as those in Experiment 1, with the following exceptions: The vibrotactile target stimuli were now presented to the same hand throughout each block of trials, whereas the distractor stimuli were always presented to the other hand. The participant was informed prior to the start of each block of trials as to which hand would receive the vibrotactile target stimuli and which hand would receive the distractors during the upcoming block. The participant was instructed to direct his/her attention covertly (i.e., while maintaining central fixation) toward the target hand. Given that target location was now fixed, we ensured that the participant maintained fixation on the central LED (rather than, say, fixating the target hand; cf. Driver \& Grossenbacher, 1996; Honoré, 1982; Honoré, Bourdeaud'hui, \& Sparrow, 1989) by monitoring the participant's eye position with an infrared camera placed directly above the fixation light. The participant's compliance with the central fixation instruction was now monitored throughout each block of experimental trials by the experimenter. If the participant's gaze was not directed straight ahead, the experimenter verbally instructed the participant to return his or her gaze to the fixation light.

The number of experimental blocks was increased from three (as in Experiment 1) to four, in order to counterbalance the side of target presentation (i.e., the participant attended to the left hand for two blocks of trials and to the right hand for the remaining two blocks of trials). The hand/side of target presentation was alternated between successive blocks of trials (half the participants started with the target presented to their left hand, and the remainder with the target presented to their right hand). The participant once again completed three practice blocks. In the first practice block, all the targets were presented to just one hand, and no distractors were presented. In the next two practice blocks, the targets were presented from just one hand, and the distractors were presented from the other hand.

\section{Results and Discussion}

Trials with an incorrect response and those with RTs outside the $\pm 2 S D$ range (4\%) were discarded from the RT analysis. A one-way ANOVA on the RT with the factor of distractor congruency revealed that the participants responded $33 \mathrm{msec}$ more slowly on incongruent trials than on congruent trials $[F(1,7)=21.4, p=.002]$ overall (see Figure 2B). Although there was a trend toward the participants' making more errors on incongruent trials than on congruent trials, this difference was not statistically significant [mean congruency effect of 3\%; $F(1,7)=2.1, p=.189]$.

Crucially, a pooled analysis using the data from Experiments 1 and 2, with experiment as the between-subjects factor and distractor congruency as the within-subjects factor, revealed that the magnitude of the congruency effect in Experiment 2 (33-msec slowing of RTs and 3\% increase in errors) was significantly smaller than that reported in Experiment 1 (173 $\mathrm{msec}$ and $16 \%$, respectively), as evidenced by the experiment $\times$ distractor congruency interactions present in both the RT and the error data $[F(1,14)=8.1, p=.013$, and $F(1,14)=10.8, p=$ .005 , respectively; compare Figures $2 \mathrm{~A}$ and $2 \mathrm{~B}]$. The participants also responded more rapidly $[F(1,14)=7.5$, $p=.016]$ and more accurately $[F(1,14)=7.1, p=.019]$, overall, in Experiment 2 than in Experiment 1.2 The main effect of congruency was also significant in terms of both the RT and the error data $[F(1,14)=17.5, p=.001$, and $F(1,14)=21.1, p<.001$, respectively]. These results are consistent with the beneficial effects of spatially focused endogenous covert attention on tactile informationprocessing reported in several previous studies (e.g., Spence, Pavani, \& Driver, 2000; Spence, Shore, \& Klein, 2001). Similar effects of spatial uncertainty have also been reported previously in many studies of selective attention within audition and vision (e.g., Chan et al., 2004; Eriksen \& Eriksen, 1974; see Styles, 1997, for a review) and by Horner (1997) in tactile pattern discrimination within a single finger.

Taken together, the results of the first two experiments therefore show that the elevation judgment task used here under conditions of unpredictable target location provided a sensitive measure of tactile selective attention between the hands. The magnitude of the interference effect reported compared favorably with that reported in previous studies (e.g., Evans \& Craig, 1991; Evans et al., 1992). In the experiments that follow, we used the elevation discrimination task under conditions of uncertain target location to address the multisensory contributions (i.e., proprioceptive and visual) to the spatial distribution of tactile selective attention.

\section{EXPERIMENT 3}

In Experiment 3, we examined whether performance on the tactile elevation discrimination task would be influenced by changes in posture, while maintaining target location fixed in terms of external space. To this end, we compared performance as the left and right hands adopted various combinations of postures: hand/wrist up (supine orientation) and hand/wrist down (prone orientation; see Figure 3). The two hands could adopt either the same orientation (i.e., both palm down, as in Experiments 1 and 2 , or both palm up) or different orientations (i.e., one hand oriented palm up and the other hand oriented palm down). When the two hands adopted the same orientation, the congruency relationship between the target and the distractor coincided in both somatotopic and external spatial coordinates. For example, when both hands were oriented palm down (as in Experiments 1 and 2), a vibration on the thumb of the target hand (bottom of the cube) was congruent with vibrations presented to the thumb of the opposite hand (bottom of the cube) and incongruent with vibrations presented to the index finger of the opposite hand (top of the cube). However, in the conditions in which the hands were placed in different orientations, the congruency relationship between the target and the distractor would no longer coincide across the different frames of reference. For example, if the target hand was oriented palm down and the distractor hand was oriented palm up, a vibration to the thumb of the target hand (bottom of the cube) would be congruent with a distractor on 


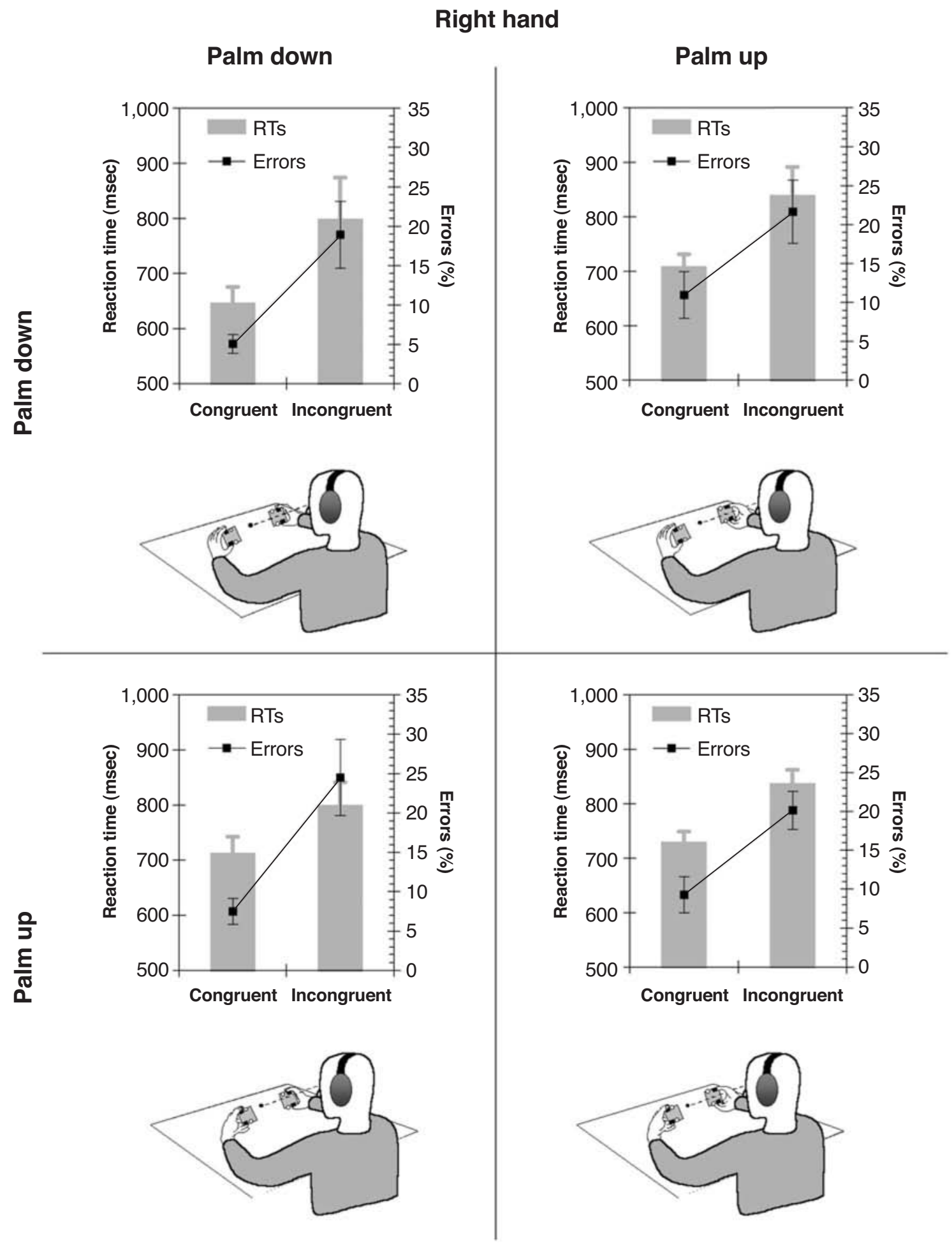

Figure 3. Results of the vibrotactile elevation discrimination task presented as a function of target-distractor congruency for the four different postures tested in Experiment 3. Below each graph is a schematic illustration of the participant's posture in that condition. In all four graphs, the bars represent the mean reaction times (RTs; see the left-hand side axis), and the filled squares connected by solid lines represent the percentages of errors (see the right-hand side axis). The error bars represent the standard errors of the means. 
the thumb in terms of somatotopic space, but incongruent in terms of externally or egocentrically defined space, since the thumb distractor vibration now occurred at the top of the cube. Similarly, with this arrangement, what would be incongruent in somatotopic terms - target at the thumb and distractor at the index finger - now would be congruent in terms of external spatial coordinates (both presented from the bottom of the target and distractor cubes).

By comparing performance in these different postures, it should be possible to determine whether congruency effects reflect spatial relationships at the level of somatotopic representations or congruency relationships operating at a more abstract (i.e., external or egocentric) representation of space (i.e., independent of the specific digits receiving the vibrotactile stimuli). Here, we chose to label the congruency conditions according to the external location of the stimuli. Thus, according to this definition, the predictions were as follows: If the same pattern of increased interference observed in the incongruent condition were to be found when the hands were positioned in different orientations, it would imply that an abstract coordinate system, independent of current hand orientation, determined the congruency effect observed. Alternatively, if the effects were reversed (i.e., the congruent trials caused more interference than the incongruent trials) in the different-orientation conditions, this would imply that congruency effects were defined in terms of a somatotopic frame of reference instead.

\section{Method}

Participants. Ten psychology students ( 5 men and 5 women) from the University of Oxford were recruited to take part in this experiment; all were naive as to the purpose of the experiment. Their mean age was 24 years (range, 19-30 years). Eight of the participants were right-handed by self-report. All reported normal tactile sensitivity and normal or corrected-to-normal vision.

Apparatus, Materials, Design, and Procedure. Given that the magnitude of the congruency effect was much larger under conditions in which target location (i.e., hand) was uncertain (Experiment 1) than when it was certain (Experiment 2), we returned to an unpredictable target location design in this and all of our subsequent experiments. The method was identical to that Experiment 1, with the exceptions noted below.

The participant completed two blocks of 20 practice trials, followed by four blocks of 64 experimental trials. The participant adopted a different posture in each block of trials. The four positions tested were both palms down (as in Experiments 1 and 2), both palms up, left palm down and right palm up, and left palm up and right palm down. In the palm-up hand position, the underside of the participant's wrist faced upward with the thumb resting on the upper vibrator placed on top of the cube, and the index finger directly below it, in contact with the bottom of the cube and the corresponding lower vibrotactile stimulator (see Figure 3). The sequence of hand orientations was alternated within subjects from block to block, whereas the starting block type was alternated between subjects. The participant was instructed to discriminate the elevation of the targets in reference to their placement on the cube (i.e., in external space, regardless of the specific digit that received the target). Note that just as in our previous experiments, the participant completed the experiment in complete darkness, so these differences in posture were signaled primarily by proprioceptive cues regarding the position of the hands. ${ }^{3}$

\section{Results and Discussion}

Trials with an incorrect response and those with RTs that fell outside the $\pm 2 S D$ range $(4 \%)$ were discarded from the RT analysis. We ran a two-way ANOVA on the RT data, including the within-subjects factors of hand orientation (both palms up, left palm up and right palm down, left palm down and right palm up, or both palms down) and distractor congruency (congruent vs. incongruent; see Figure 3). This analysis revealed a significant main effect of distractor congruency $[F(1,9)=12.8, p=$ $.006]$, with participants responding $119 \mathrm{msec}$ more quickly on congruent trials than on incongruent trials overall. There was no main effect of hand orientation $(F<1)$ or any interaction between distractor congruency and hand orientation $[F(3,27)=1.0, p=.392]$. The analysis of the error data revealed the same pattern of results, with a significant main effect of distractor congruency [mean congruency effect of $13 \% ; F(1,9)=11.9, p=.007]$, but no main effect of hand orientation $[F(3,27)=1.4, p=$ $.254]$ or any interaction between these two factors $(F<1)$.

In this experiment, the position of the stimuli was fixed in terms of external space but varied in terms of somatotopic space across the different hand postures tested. Yet stimulation to a particular skin location (finger) adopted a different valence within the task, depending on the external location in which it was placed (i.e., top vs. bottom of the cube). The failure of hand orientation to modulate the direction or magnitude of the intramodal tactile congruency effect demonstrated that the reference frame determining interference effects cannot be based solely on somatotopic coordinates. Instead, it appears that the location of vibrotactile stimuli must be represented in a more abstract frame of reference, possibly defined by the position of the stimuli in external space or relative to some part of the body (i.e., head or trunk).

Overall, our results appear to converge with Rinker and Craig's (1994) previous findings, using a motion discrimination task, while extending them from selection occurring within one hand (as in all previous studies in which the effects of rotation of the hand were looked at; e.g., Moscovitch \& Behrmann, 1994; Rinker \& Craig, $1994)$ to the selection of stimuli taking place across the hands. Note, however, that the present pattern of results was obtained when the participant's task was to respond to the elevation of the targets (i.e., in external coordinates). It will be an interesting question for future research to examine whether a similar pattern of results would be found if the task were altered so that the participants were required to respond on the basis of the somatotopically defined location of the targets instead (i.e., by making one response to targets delivered to the index finger and another response to targets delivered to the thumbs, regardless of the posture adopted-i.e., palm 
up or palm down; cf. Austen, Soto-Faraco, Enns, \& Kingstone, 2004).

Having demonstrated that variations in the somatotopic location from which vibrotactile stimuli are presented have no effect on performance as long as congruency relationships are kept fixed in external (and egocentric) space, we next evaluated the consequences of varying the separation between the hands in external space while keeping the somatotopic location of tactile stimuli (and hand orientation) fixed. As was discussed in the introduction, two of the most important cues regarding the posture of the body come from proprioception and vision. In the next two experiments, we evaluated the role of each of these cues in modulating the intramodal tactile congruency effect.

\section{EXPERIMENT 4}

In Experiment 4, we compared the magnitude of the congruency effect observed when the target and the distractor hands held the cubes close together ( $5 \mathrm{~cm}$ apart) versus far apart (100 cm apart). If, as was suggested by the results of Experiment 3, tactile selective attention operates in an abstract frame of reference (be it externally or egocentrically based), the interference effect produced by the distractors should diminish as distractor distance in external space increases (e.g., Driver \& Grossenbacher, 1996; Lakatos \& Shepard, 1997). If, however, it is only somatotopic distance that matters, equivalent results should be reported in both conditions (i.e., no matter whether the hands are positioned close together or far apart), since distance in somatotopic space is unaffected by any change in posture (as was reported by Evans et al., 1992). In the present experiment, we introduced a difference of about $95 \mathrm{~cm}$ between the hands-near and the hands-far conditions, in order to maximize our chances of finding a spatial modulation of tactile spatial attention. Indeed, there is a possibility that the use of only a small difference in hand separation $(32 \mathrm{~cm})$ may have explained the null results reported by Evans et al., in contrast with the significant interaction reported by Driver and Grossenbacher (1996, who used a hand separation of $66 \mathrm{~cm}$ ). Note that because our experiment was conducted in darkness, the participants had continued direct access only to proprioceptive cues regarding the position of their hands in space.

\section{Method}

Participants. Ten psychology students ( 3 men and 7 women) from the University of Oxford were recruited to take part in the experiment; all were naive as to the purpose of the experiment. Their mean age was 20 years (range, 19-21 years). Seven of the participants were right-handed by self-report. All reported normal tactile sensitivity and normal or corrected-to-normal vision.

Apparatus, Materials, Design, and Procedure. The apparatus, materials, design, and procedure were exactly the same as those in Experiment 1, with the following exceptions. In the hands-near condition, the participants held the two cubes $5 \mathrm{~cm}$ from each other (centered on the midline), whereas in the hands-far condition, the participants held the cubes $100 \mathrm{~cm}$ apart (again centered on the midline; see Figure 4). The participants performed three blocks of practice trials, followed by four test blocks of 64 trials. The position of the hands was changed after every block of trials so that the hands-near and hands-far conditions alternated (with the order of presentation counterbalanced across participants).

\section{Results and Discussion}

Trials with an incorrect response and those with RTs outside the $\pm 2 S D$ range (5\%) were discarded from the RT analysis. An ANOVA was conducted on the RT data with the within-subjects factors of hand separation (hands near vs. hands far) and distractor congruency (congruent vs. incongruent). As in all the previous experiments, there was a significant main effect of distractor congruency $[F(1,9)=13.6, p=.005]$, with participants responding significantly more quickly (by $143 \mathrm{msec}$ ) on congruent trials (in which the elevation of the distractor was the same as the elevation of the target) than on incongruent trials (in which the elevation of the distractor was different from the elevation of the target). However, this analysis did not reveal a main effect of hand separation $(F<1)$ or any interaction between hand separation and distractor congruency $(F<1$; see Figure 4$)$.

The analysis of the error data also revealed a significant main effect of distractor congruency $[F(1,9)=47.8$, $p<.001$ ], with participants making $12 \%$ more errors on incongruent trials than on congruent trials overall. The participants tended to make more errors in the hands-near condition than in the hands-far condition $(12 \%$ vs. $10 \%$, respectively), although this effect just failed to reach statistical significance $[F(1,9)=4.4, p=.066]$. Importantly, however, there was a significant interaction between distractor congruency and hand separation $[F(1,9)=7.8, p=$ .021 ], attributable to the larger congruency effect reported when the hands were placed close together $(15 \%)$ than when they were placed far apart $(10 \%)$. The congruency effect was, nevertheless, significant in both postures $[t(9)=$ $6.8, p<.001$, and $t(9)=5.4, p<.001$, respectively].

Increasing the distance between the target and the distractor in external space while maintaining distance constant in terms of somatotopic space resulted in a significant reduction in the magnitude of the congruency effect, as reflected by the error data. This result is contrary to what one would expect if the spatial frame of reference operating during tactile selective attention was based strictly on a somatotopic coordinate system. The results of Experiment 4, together with the data obtained in Experiment 3, therefore confirm the view that spatial attention in touch is based on an abstract coordinate system (either egocentrically or externally based) that is updated as a result of proprioceptive inputs.

In the experiments reported so far, the participants received no direct visual cues regarding the posture of their body, since all of the experiments were conducted in complete darkness. This ensured that information about body posture was obtained mainly via proprioceptive cues, since information was continuously available in 


\section{Experiment 4}

(A) Hands close
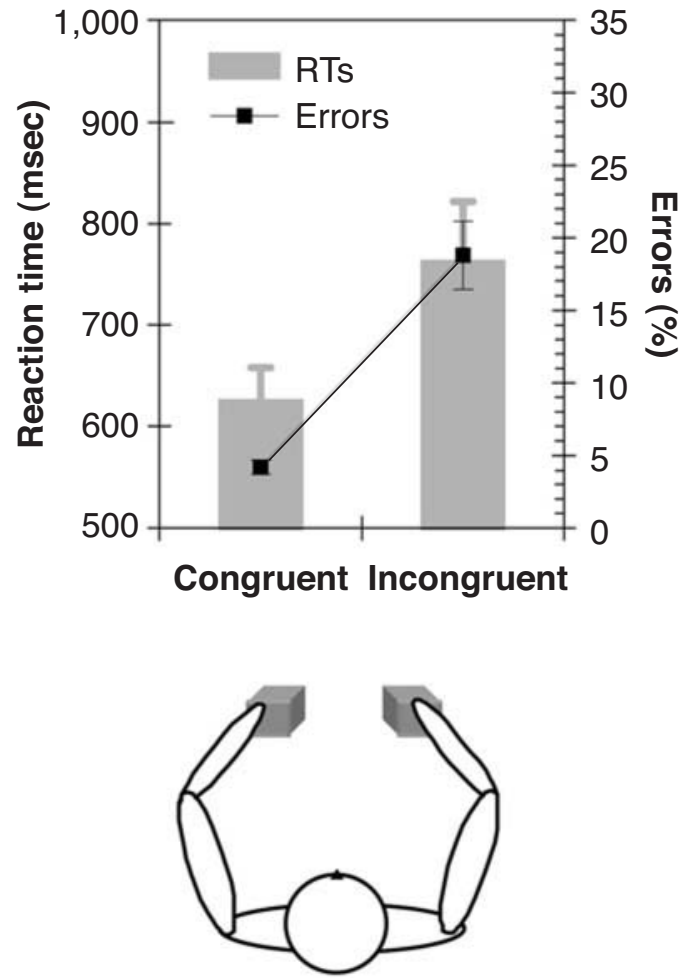

(B) Hands far
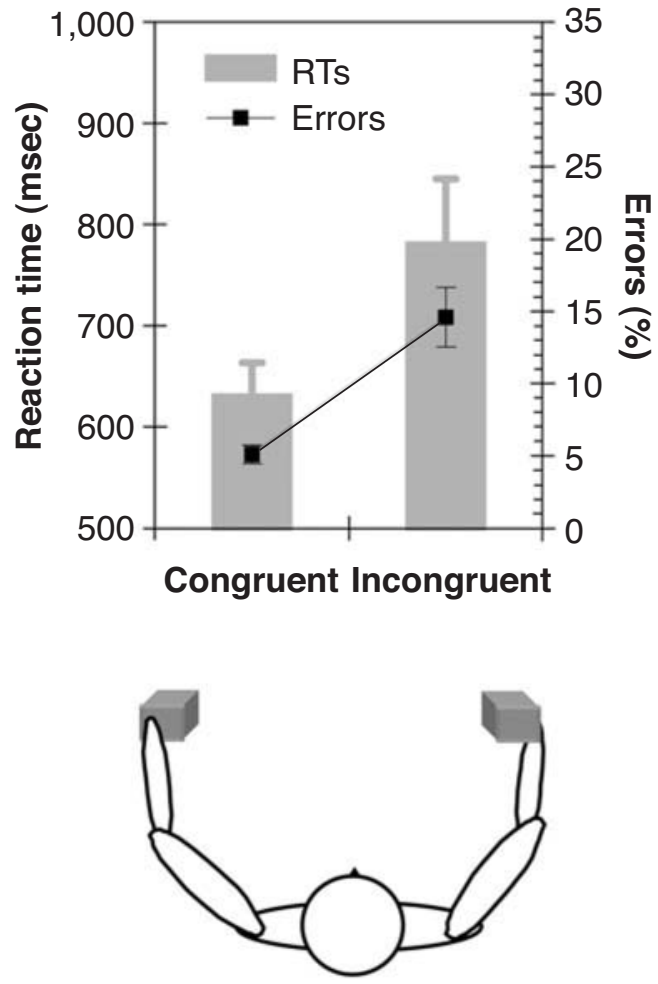

Figure 4. Results of the vibrotactile elevation discrimination task as a function of target-distractor congruency and an outline of the different body postures adopted by the participants in Experiment 4 . The bars represent the mean reaction times (RTs; see the left-hand side axis). The error bars represent the standard errors of the means. The square symbols connected by the solid lines represent the percentages of errors (see the right-hand side axis). The error bars represent the standard errors of the means. Graph A shows performance when the cubes were held close together $(5 \mathrm{~cm})$, and Graph B shows performance when the cubes were held far from each other $(100 \mathrm{~cm})$.

this modality. However, in many everyday circumstances, proprioception is not the only source of information regarding limb position, since visual cues also play an important role in determining where we perceive our limbs to be. Unfortunately, there is no simple way by which one can eliminate proprioceptive cues in order to assess the net contribution of visual cues alone on the spatial frame of reference during tactile attention (but see Cole, 1991, for studies of a patient suffering from a chronic loss of proprioception attributable to pathological deafferentation). Therefore, in Experiment 5, we assessed the effects of visual information about body posture by placing visual and proprioceptive cues into conflict instead.

Research has shown that when visual and proprioceptive cues regarding posture are put into conflict artificially (by means of the use of mirrors, video feedback, or artificial body parts), vision often dominates in terms of the final percept (e.g., Austen et al., 2004; Botvinick \& Cohen, 1998; Maravita, Spence, Clarke, Husain, \& Driver, 2000; Maravita, Spence, Sergent, \& Driver, 2002;
Nielsen, 1963; Pavani, Spence, \& Driver, 2000; Tastevin, 1937). However, in all the previous experiments on selective tactile attention, either only proprioceptive cues (e.g., vision was prevented altogether) or combined congruent proprioceptive and visual cues (e.g., vision was allowed, and proprioception and vision provided correlated postural information) have been used. In our final experiment, we studied the contribution of visual feedback to the maintenance of the spatial representation upon which tactile attention operates.

\section{EXPERIMENT 5}

In Experiment 5, proprioceptive cues regarding limb position were held constant (by keeping the hands at a fixed position and distance), while the apparent separation of the arms was manipulated by the use of visual cues regarding limb position elicited via a mirror reflection. By comparing the magnitude of any congruency effects when the hands appeared "visually" to be close to- 
gether versus when the hands were visibly far apart, we hoped to determine whether any visual capture of perceived limb position would also modulate the magnitude of the tactile congruency effects observed. Note that although previous studies have shown that, in some circumstances, visual cues can dominate over proprioceptive cues regarding the perception of body posture, this pattern of dominance is not necessarily such an obvious result when the task solely involves tactile selective attention. For example, Kennett et al. (2002) reported some evidence for an increased role of proprioceptive cues in modulating cross-modal shifts of exogenous spatial attention following posture change (such as crossing the hands) when participants were required to perform a vibrotactile, as opposed to a visual, discrimination task (see also Spence \& Walton, 2004).

In our final experiment, the participant placed the left hand close to the body midline and extended the right hand away from their trunk (so that the distance between the hands was as large as that in the hands-far condition of Experiment 4). This posture was maintained throughout the entire experiment. In half of the blocks of trials, a mirror was placed perpendicular to the participant's chest, with its reflective surface facing the participant's left hand and the participant's right hand being occluded from view behind the mirror (see Figure 5). Under these conditions, the reflection of the left hand in the mirror appeared to be the right hand, placed close to the left hand a few centimeters across the midline. If proprioceptive cues provide the major source of information regarding limb position, the visual feedback coming from the mirror should have had little effect on the pattern of performance observed. Hence, no difference in the magnitude of the congruency effects would be predicted as a function of whether the mirror was present or not. However, if visual input plays a role in the construction of the

\section{Experiment 5}

(A) Hands visibly "close"
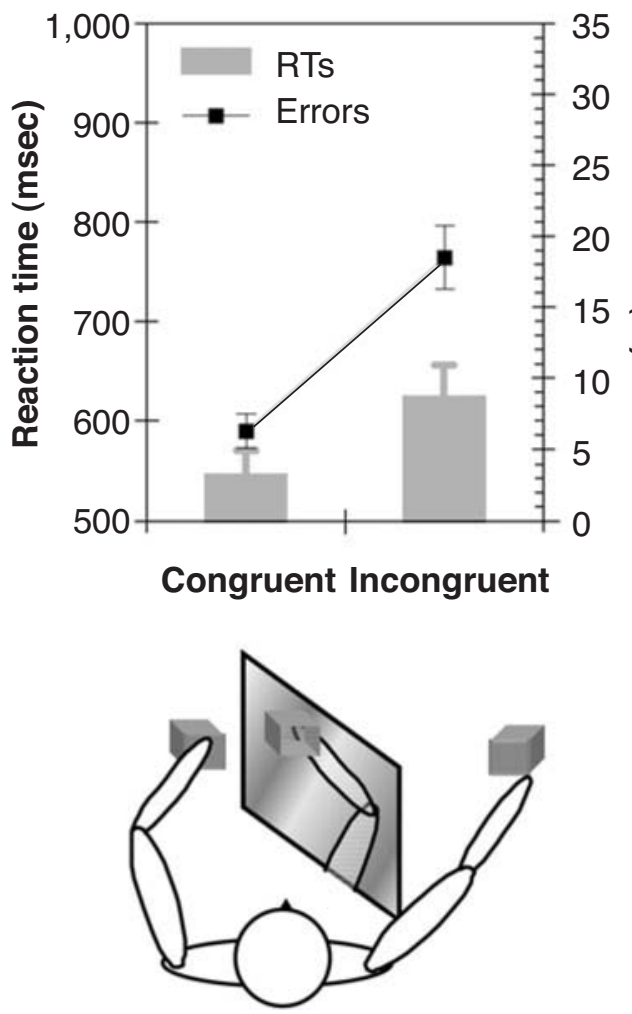

(B) Hands visibly "far"
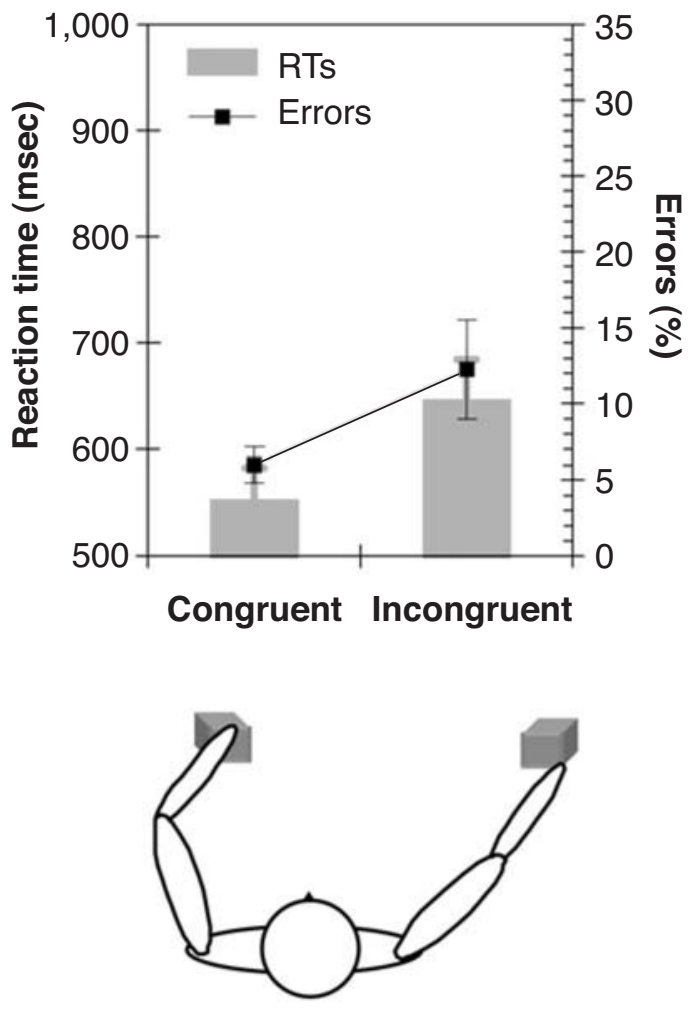

Figure 5. Results of the vibrotactile elevation discrimination task as a function of target-distractor congruency and a schematic outline of the different set-ups used in Experiment 5. The bars represent the mean reaction times (RTs; see the left-hand side axis). The square symbols connected by the solid lines represent the percentages of errors (see the right-hand side axis). The error bars represent the standard errors of the means. Graph A shows performance when the cubes appeared to be close together (due to the participant's seeing the mirror reflection of the left hand as if it were the right hand) but were actually held far from each other, and Graph B shows performance when the cubes were both seen and felt to be held far from each other. 
spatial representation in which tactile stimuli are located during selective attention, the congruency effect observed in the presence of the mirror should increase to a magnitude similar to that found when the hands are actually placed physically close together (as reported in Experiment 4).

\section{Method}

Participants. Eight psychology students (4 men and 4 women), all naive as to the purpose of the experiment, were recruited to take part. Their mean age was 20 years (range, 18-22 years), and none of them had participated in any of the previous experiments reported in this manuscript. Six of the participants were right-handed by self-report. All reported normal tactile sensitivity and normal or corrected-to-normal vision.

Apparatus, Materials, Design, and Procedure. The apparatus, materials, design, and procedure were exactly the same as those in Experiment 4, with the following exceptions. The participant's left hand was now placed $2 \mathrm{~cm}$ to the left of the participant's midline, while the right hand was placed approximately $95 \mathrm{~cm}$ to the right of the midline (see Figure 5). The participant maintained this posture throughout every block of trials. In the near condition, a mirror was positioned on an almost vertical plane perpendicular to the participant's chest, with the reflective surface facing to the left. When the participant looked at the fixation light, the left hand appeared to be approximately $5 \mathrm{~cm}$ away from its reflection in the mirror, which looked in appearance and posture as if it were the "right" hand holding the second foam cube. A black cloth suspended above and behind the mirror prevented the participant from seeing the actual position of the right hand far away behind the mirror. Prior to the start of each block of test trials in the near condition, the participant was instructed to move his or her hands in synchrony in order to establish the visual capture effect. ${ }^{4}$ In the far condition, the mirror and black cloth were simply removed so that the participant could see the right hand resting on the table far from the left hand. Experiment 5 was the only experiment in which the room was illuminated by the ceiling lights (in order to allow the participant to see the mirror reflection of the left hand). The near and far conditions were alternated (two blocks of each for a total of four blocks), with half the participants beginning with the near condition and the other half with the far condition.

\section{Results and Discussion}

Trials with an incorrect response and those with an RT outside the $\pm 2 S D$ range (5\%) were discarded from the RT analysis. The ANOVA on the RT data included the within-subjects factors of apparent hand separation (hands near vs. hands far) and distractor congruency (congruent vs. incongruent). As in the previous experiments, there was a significant main effect of distractor congruency $[F(1,7)=16.1, p=.005]$, attributable to the fact that the participants responded $86 \mathrm{msec}$ faster on congruent trials than on incongruent trials overall. There was no significant main effect of apparent hand separation on performance $[F(1,7)=2.3, p=.172]$ or any interaction between the apparent hand separation and the distractor congruency factors $(F<1$; see Figure 5$)$.

A similar analysis on the error data again revealed a significant main effect of congruency [mean congruency effect of $9 \% ; F(1,7)=13.2, p=.008]$. Apparent hand separation also reached significance; when the hands appeared to be situated close together (because of the visual cue provided by the mirror reflection of the right hand), the participants made 3\% more errors than when the participants could see their hands to be far apart [i.e., in the no-mirror condition; $F(1,7)=14.1, p=.007]$. Critically (and just as in Experiment 4), the interaction between apparent hand separation and distractor congruency was also significant $[F(1,7)=6.6, p=.037]$, reflecting the fact that larger congruency effects were reported when the hands appeared to be close together visually than when the mirror was removed and the hands were seen to be in their actual position, far from one another (mean congruency effects of $12 \%$ and $6 \%$, respectively). ${ }^{5}$

Despite of the fact that the participants' hands always remained in the same location (i.e., far from each other), the interference effect elicited by vibrotactile distractors increased (as in Experiment 4, in terms of the accuracy data) when the hands appeared visually to be close together, due to the mirror reflection. Interestingly, this visual modulation of tactile selective attention occurred despite the fact that vision was entirely irrelevant to the participant's task. Other researchers have obtained similar results, using a cross-modal version of this congruency effect, in which the distractors are presented visually (i.e., Maravita et al., 2002; Pavani et al., 2000). However, in contrast to these previous studies, the visual input in the present study consisted solely of a fixed scene that did not contain any attention-grabbing transient visual events (that might have captured the participants' visual attention momentarily while they were trying to respond to the vibrotactile targets). It would appear, then, that this multisensory integration of visual, proprioceptive, and tactile information took place automatically, given that the occurrence of the mirror illusion was actually detrimental to performance (and so the participants would have been well advised to try and prevent any such integration of visual cues with proprioceptive and tactile information, if at all possible).

\section{GENERAL DISCUSSION}

One of the most consistent results to emerge from the analysis of the five experiments reported in the present study is that participants cannot ignore the elevation of a pulsed vibrotactile distractor presented to one hand while making speeded elevation discrimination responses to continuous vibrotactile targets presented to the other hand. Significant intramodal tactile congruency effects were reported in the RT data from every experiment and from all the experiments except Experiment 2 (when target side was made predictable) in terms of the accuracy data. Our results therefore support previous studies demonstrating interference effects when tactile target and distractor stimuli are presented to different hands (e.g., Driver \& Grossenbacher, 1996; Evans et al., 1992). ${ }^{6}$ With this new version of the bimanual interference effect, several additional findings also emerged. First, spatial uncertainty concerning which hand would receive the target resulted 
in a dramatic increase in the interference effects of irrelevant distractors. Second, tactile spatial attention can operate within a coordinate system based on an abstract (egocentrically or externally defined) frame of reference, which is updated as new postures are adopted. This result holds, at least, when the participant's task is to respond on the basis of the externally determined spatial properties of the stimuli, as in the present study. Finally, we were able to show that both proprioception and vision make an important contribution to the construction of this abstract frame of reference. In the following, we will discuss the implications of these results.

\section{The Effects of Spatial Uncertainty on Tactile Selective Attention}

When the participants were able to direct their attention selectively to the target hand before target onset (i.e., when the target and the distractor stimuli were each predictably presented from a different hand throughout each block of trials; Experiment 2), distractor congruency effects were reduced significantly, as compared with when their location of presentation was unpredictable (Experiment 1). It is interesting to note here that in Experiment 2, the congruency effects were still significant, albeit reduced, in terms of the RT data but that they no longer reached significance in terms of accuracy data, precisely the dependent measure used by Evans et al. (1992, Experiment 3) in a study that failed to show any interference across different hands. Thus, from our results, it appears that people are capable of directing their spatial attention to a particular hand (although not perfectly, as is shown by the significant interference found in the predictable location conditions of Experiment 2) and, by so doing, can facilitate the processing of target stimuli presented there, relative to stimuli presented to the other hand (see Spence, Pavani, \& Driver, 2000, for similar results regarding tactile selective attention based on a Posnerian spatial-cuing design). The focused attention conditions of Experiment 2 led to shorter average response latencies than did those reported in Experiment 1, suggesting that shifting attention across the body surface to detect target stimuli takes time. This finding is also in accord with previous studies addressing tactile attention shifts across the body surface (e.g., Lakatos \& Shepard, 1997) and with the attention-shifting costs found with other sensory modalities (i.e., Mondor \& Zatorre, 1995).

\section{Spatial Modulation of Tactile Selective Attention}

In Experiment 3, we manipulated spatial relationships in terms of somatotopic coordinates while keeping spatial relationships fixed in terms of external (as well as head- and trunk-centered) coordinates. We showed that the tactile congruency effect for spatial elevation discrimination judgments was determined not by the relative locations of the target and distractor stimuli on the somatotopically arrayed body surface (i.e., finger vs. thumb), but by the relative position of the target and the distractor stimuli in external space (i.e., top vs. bottom).
This result, which was obtained under bimanual stimulation conditions, is consistent with previous findings when a direction of motion discrimination task was used under unimanual presentation conditions (Rinker \& Craig, 1994). Our results also converge with data from neuropsychological studies showing that the syndrome of tactile extinction seems to operate on an abstract (egocentrically or externally defined) frame of reference in brain lesion patients (Aglioti et al., 1999; Moscovitch \& Behrmann, 1994; Tinazzi et al., 2000).

In Experiment 4, we demonstrated a modulation of the tactile interference effect by target-distractor separation between the hands in terms of external space while keeping somatosensory distance fixed. In particular, the extent to which a vibrotactile distractor will interfere with discrimination responses for vibrotactile targets depends on the relative separation of the two hands in external space: The closer the distractor is to the target, the harder it is for people to ignore it. Similar spatial modulations of congruency (or flanker interference) effects have been reported within other sensory modalities, such as audition and vision (e.g., Chan et al., 2004; Eriksen \& Eriksen, 1974; see Styles, 1997, for a review), and also in numerous previous studies of intramodal tactile selective attention (e.g., Craig, 1974; Gilson, 1969; Weisenberger, 1994; Weisenberger \& Craig, 1982). However, these earlier studies often manipulated spatial distance in somatotopic and abstract reference frames at the same time, and therefore, the question of which frame of reference is relevant during tactile spatial selective attention remained unanswered. Our results with the elevation discrimination task coincide with those of more recent studies in which different methods were used (Driver \& Grossenbacher, 1996; Lakatos \& Shepard, 1997) and, when taken together with the results of Experiment 3, allow us to demonstrate that the frame of reference that subserves tactile spatial-selective attention is not defined solely in terms of somatotopic coordinates, but that a more abstract frame of reference can also be used.

However, it must be noted that the magnitude of the effect of hand separation in Experiment 4 was small. Indeed, only the accuracy data revealed a significant interaction between posture and spatial congruency. This may be a sign that the participants in this experiment prioritized response speed over response accuracy, but it may also suggest that the effect itself was quite a weak one. This might very well explain why the effects of hand separation have sometimes been shown to be very sensitive to the particular experimental parameters used (such as the magnitude of hand separation or the task used) in previous studies. Indeed, Evans et al. (1992) failed to observe any interaction in terms of accuracy (they did not measure response latencies in their experiment), contrasting with the already mentioned findings of Driver and Grossenbacher (1996). In the present Experiment 4, we found that there is a small but significant effect in terms of accuracy (which was replicated under similar conditions in Experiment 5). 
One important feature of the design of both Experiments 3 and 4 is that we ensured that cues to body posture (i.e., signaling the distance between the hands) were primarily proprioceptive, since visual information was prevented by running the experiments in darkness. In Experiment 5, we took this hand separation effect one step further by demonstrating that even virtual changes in posture elicited by the use of visual cues to body posture via a mirror reflection could also modulate the magnitude of the interference (see Burnett, 1904, for an early application of the mirror illusion, and Maravita et al., 2002, for a more recent example). Here, we kept somatosensory distance fixed (as in Experiment 4), but we also kept proprioceptive cues to body posture constant across conditions, while varying only visual cues to body posture. The main finding to emerge from this manipulation was that, by themselves, visual cues to hand posture are enough to produce a modulation of tactile selective attention (perhaps via the capturing of the proprioceptive sense of hand position).

This visual capture effect elicited by the mirror manipulation bears more than a passing resemblance to the mirror box used by some researchers in their attempt to relieve the pain that can be associated with the experience of a phantom limb in amputees (e.g., Ramachandran \& Rogers-Ramachandran, 1996; Ramachandran, RogersRamachandran, \& Cobb, 1995; Sathian, Greenspan, \& Wolf, 2000). In both cases, the visual image of one arm seen in a mirror elicits a change (or visual capture) of the perceived location of the other hand/arm (be it real, as in our study, or virtual, as for phantom limb patients). For some phantom limb patients, the extended use of the mirror box has been reported to alleviate temporarily the painful sensations associated with the phantom. ${ }^{7}$ In the present study, this visual capture effect modulated the magnitude of tactile congruency effects (see also Nielsen, 1963, for related findings in normal participants).

\section{Multisensory Integration Prior to Tactile Selective Attention}

As has been noted, a vibrotactile stimulus by itself (in the absence of information about the posture of the body) cannot be unambiguously localized in external space or with respect to some body parts (such as the head or the trunk). Instead, tactile information must be integrated with information from the other senses, such as proprioception and vision, in order for touch to be localized correctly in the environment. Our results suggest that this multisensory integration leading to the localization of tactile stimuli with respect to our body and/or in external space must take place prior to the operation of tactile selective attention. This implication contrasts with traditional views of attention in which it has been argued that feature integration requires attention (e.g., Treisman, 1998; Treisman \& Gelade, 1980). However, because attention operates within an abstract spatial frame of reference, tactile information (initially encoded somatotopically) must have been combined with information from other sensory modalities (such as proprioception and vision) prior to (and not as a consequence of) attentional selection. The idea that multisensory integration can occur prior to attentional selection has obtained increasing empirical support from recent multisensory research on audiovisual interactions (e.g., see the chapters in Spence \& Driver, 2004). The results of Experiment 4 suggest that the same applies to the integration of proprioceptive and tactile information, and Experiment 5 extends this argument to the case of visual-proprioceptive integration as well.

\section{Intramodal Versus Cross-Modal Congruency Effects: Similarities and Differences}

A growing body of research from this laboratory (e.g., Maravita et al., 2002; Pavani et al., 2000; Spence, Kingstone, Shore, \& Gazzaniga, 2001; Spence, Shore, Gazzaniga, Soto-Faraco, \& Kingstone, 2001) has used a cross-modal version of the present congruency task, in which participants are required to discriminate the elevation of a vibrotactile target presented to the thumb or index finger of one or the other hand, while simultaneously trying to ignore visual distractors that can be presented from the same or a different elevation (instead of the pulsed tactile distractors used in the present study). The close similarity between the two sets of results suggests that these interactions may obey similar rules within and across different sensory modalities. Interestingly, the overall magnitude of these congruency effects seems to be even greater in those previous cross-modal studies of selective attention than in the intramodal studies of selective attention reported here. This is contrary to what is usually shown in other paradigms, where cross-modal effects are often weaker than the corresponding intramodal effects (see Eimer \& Schröger, 1998; Mangun, Hillyard, \& Luck, 1993; Spence \& Driver, 1996, 1997). It is uncertain, however, whether this reflects differences in the efficacy of intramodal versus cross-modal selection or, alternatively, just differences in the relative saliency of the distractors used in the various studies. Crucially, however, both intramodal tactile and cross-modal visuotactile selective attention effects show spatial modulation dependent on the relative location of targets and distractors. What's more, both effects are also sensitive to virtual changes in posture elicited by the use of either rubber hands or mirrors (see the present Experiment 5; see also Austen et al., 2004; Maravita et al., 2002; Pavani et al., 2000).

\section{Comparison With Effects of Posture Change in Other "Tactile" Tasks}

In the last few years, a growing body of research has demonstrated the dramatic effects of posture change on tactile spatial localization (e.g., Craig \& Busey, 2003; Eimer, Cockburn, Smedley, \& Driver, 2001; Shore, Spry, \& Spence, 2002; Spence, Harris, \& Zampini, 2003; Yamamoto \& Kitazawa, 2001). For example, Shore et al. showed a strong accuracy decrement in temporal order 
judgments regarding two tactile stimuli delivered one to either hand when participants adopted a crossed hands posture (see also Yamamoto \& Kitazawa, 2001). More recently, Craig and Busey (2003) have shown that the influence of the tactile motion of stimuli on judging which of two tactile patterns was presented first is also modulated by changes in posture. Interleaving the fingers of the two hands can also influence tactile perception, as in the so-called Aristotle illusion (e.g., Benedetti, 1985, 1988; Ponzo, 1910) and possibly also in the Japanese illusion (Henri, 1898; Spence et al., 2003). Eimer et al. also found a consistent change (decrement) in the electrophysiological correlates of tactile spatial attention when the hands were placed in a crossed, as opposed to an uncrossed, posture.

These effects have typically been observed under conditions in which participants could not see their limbs directly, and as such, probably reflect the consequences of change signaled proprioceptively (rather than visually). Such results converge with the present findings in that they also suggest that as the body adopts different postures, there is a remapping of tactile spatial information according to information from muscle position and gravitational forces to locate tactual stimuli accurately in space. However, it seems that sometimes, this remapping is far from perfect, and it may take some time to build up an appropriate representation of the body and its limbs in space $^{8}$ (e.g., Eimer et al., 2001; Kitazawa, 2002; Yamamoto \& Kitazawa, 2001). Clearly, this abstract frame of reference provides a candidate subserving the coordination between selective attention in touch and in other sensory modalities (see Eimer et al., 2001; Kennett et al., 2001; Spence, 2001).

\section{Future Directions}

There are a number of challenging questions to be addressed by future research. For example, we still do not have a clear answer to the question of why some studies have shown effects in spatial tactile attention that seem to respond solely to a somatotopic frame of reference (Röder et al., 2000), whereas others, like the present study, clearly have shown that tactile attention can operate in a more abstract coordinate system (based in egocentrically or externally based spatial frame of reference). One speculative account could be based on the different attention mechanisms involved. Röder et al.'s study involved exogenous (or reflexive) attentional mechanisms (in particular, the phenomenon of IOR), whereas the studies showing that tactile attention operates in an external frame of reference have typically involved endogenous (or voluntary) mechanisms of attention (see Driver \& Spence, 1998b, and Klein \& Shore, 2000, on this issue). For this distinction to work, one would need to accept that Evans and Craig's (1991) failure to find interference effects across the hands and Evans et al.'s (1992) failure to find an effect of hand separation can be explained by methodological aspects of the experimental designs used (as was discussed earlier). Even then, this distinction may not be as clear-cut as one might like. For, as some authors have proposed, both types of reference frames could coexist during selective attention (and consequently, in perception), and sometimes they may even provide conflicting information under certain conditions (e.g., Eimer et al., 2001; Kim \& Cruse, 2001; Ponzo, 1910).

\section{REFERENCES}

Aglioti, S., Smania, N., \& Peru, A. (1999). Frames of reference for mapping tactile stimuli in brain-damaged patients. Journal of Cognitive Neuroscience, 11, 67-79.

Austen, E. L., Soto-FARAco, S., Enns, J. T., \& Kingstone, A. (2004). Mislocalizations of touch to a fake hand. Cognitive, Affective, \& Behavioral Neuroscience, 4, 170-181.

BenedetTI, F. (1985). Processing of tactile spatial information with crossed fingers. Journal of Experimental Psychology: Human Perception \& Performance, 11, 517-525.

BenedetTI, F. (1988). Exploration of a rod with crossed fingers. Perception \& Psychophysics, 44, 281-284.

Botvinick, M., \& Cohen, J. (1998). Rubber hands "feel" touch that the eyes see. Nature, 391, 756.

BRIGNER, W. L. (1984). Rotation of space-time plane predicts a new illusion of spatial displacement. Perceptual \& Motor Skills, 59, 359369.

BurnetT, C. T. (1904). Studies on the influence of abnormal position upon the motor impulse. Psychological Review, 11, 370-394.

Chan, J. S., Merrifield, K., \& Spence, C. (2004). Auditory spatial attention assessed in a flanker interference task. Manuscript submitted for publication.

CoLe, J. (1991). Pride and a daily marathon. London: Duckworth.

Craig, J. C. (1974). Vibrotactile difference thresholds for intensity and the effect of a masking stimulus. Perception \& Psychophysics, $\underline{\mathbf{1 5}}$, 123-127.

CRAIG, J. C., \& Busey, T. A. (2003). The effect of motion on tactile and visual temporal order judgments. Perception \& Psychophysics, $\underline{\mathbf{6 5}}$, 81-94.

DrIVER, J., \& Grossenbacher, P. G. (1996). Multimodal spatial constraints on tactile selective attention. In T. Inui \& J. L. McClelland (Eds.), Attention and performance XVI: Information integration in perception and communication (pp. 209-235). Cambridge, MA: MIT Press.

Driver, J., \& SPENCE, C. (1998a). Attention and the crossmodal construction of space. Trends in Cognitive Sciences, 2, 254-262.

Driver, J., \& SPEnCE, C. (1998b). Crossmodal links in spatial attention. Philosophical Transactions of the Royal Society of London: Series B, 353, 1319-1331.

Eimer, M., CockBurn, D., Smedley, B., \& Driver, J. (2001). Crossmodal links in endogenous spatial attention are mediated by common external locations: Evidence from event-related brain potentials. Experimental Brain Research, 139, 398-411.

EIMER, M., \& SCHRÖGER, E. (1998). ERP effects of intermodal attention and cross-modal links in spatial attention. Psychophysiology, 35, 313-327.

ERIKSEN, B. A., \& Eriksen, C. W. (1974). Effects of noise letters upon the identification of a target letter in a nonsearch task. Perception \& Psychophysics, 16, 143-149.

Evans, P. M., \& Craig, J. C. (1991). Tactile attention and the perception of moving tactile stimuli. Perception \& Psychophysics, 49, 355-364.

Evans, P. M., Craig, J. C., \& Rinker, M. A. (1992). Perceptual processing of adjacent and nonadjacent tactile nontargets. Perception \& Psychophysics, 52, 571-581.

GILSON, R. D. (19 $\overline{69})$. Vibrotactile masking: Some spatial and temporal aspects. Perception \& Psychophysics, 5, 176-180.

Henri, V. (1898). Über die Raumwahrnehmung des Tastsinnes: Ein Beitrag zur experimentellen Psychologie [On the spatial perception of the tactile sense: A contribution to experimental psychology]. Berlin: Reuther \& Reichard. 
Holmes, N. P., Crozier, G., \& Spence, C. (2004). When mirrors lie: "Visual capture" of arm position impairs reaching performance. Cognitive, Affective, \& Behavioral Neuroscience, 4, 193-200.

HONORÉ, J. (1982). Posture oculaire et attention selective a des stimuli cutanes [Eye position and selective attention to cutaneous stimuli]. Neuropsychologia, 20, 727-730.

HonORÉ, J., BourdeaUd'HUI, M., \& SPARrow, L. (1989). Reduction of cutaneous reaction time by directing eyes towards the source of stimulation. Neuropsychologia, 27, 367-371.

HorNer, D. T. (1995). The effect of location on the discrimination of spatial vibrotactile patterns. Perception \& Psychophysics, 57, 463-474.

HoRner, D. T. (1997). The effect of shape and location on temporal masking of spatial vibrotactile patterns. Perception \& Psychophysics, 59, 1255-1265.

HORNER, D. T. (2000). Perceptual processing at adjacent locations on a single finger: Masking and response competition. Perception \& Psychophysics, 62, 1545-1555.

JoNES, F. N. (1956). Space-time relationships in somesthetic localization. Science, 124, 484.

Kennett, S., Eimer, M., SPence, C., \& Driver, J. (2001). Tactile-visual links in exogenous spatial attention under different postures: Convergent evidence from psychophysics and ERPs. Journal of Cognitive Neuroscience, 13, 462-478.

KenNett, S., SPEnCE, C., \& Driver, J. (2002). Visuo-tactile links in covert exogenous spatial attention remap across changes in unseen hand posture. Perception \& Psychophysics, 64, 1083-1094.

KIM, D. H., \& CRUSE, H. (2001). Two kinds of body representation are used to control hand movements following tactile stimulation. Experimental Brain Research, 139, 76-91.

KitAZAWA, S. (2002). Where conscious sensation takes place. Consciousness \& Cognition, 11, 475-477.

KLEIN, R. M. (2000). Inhibition of return. Trends in Cognitive Sciences, 4, 138-147.

KLEIN, R. M., \& SHORE, D. I. (2000). Relationships among modes of visual orienting. In S. Monsell \& J. Driver (Eds.), Control of cognitive processes: Attention and performance XVIII (pp. 195-208). Cambridge, MA: MIT Press.

LAKatos, S., \& Shepard, R. N. (1997). Time-distance relations in shifting attention between locations on one's body. Perception \& Psychophysics, 59, 557-566.

MANGUN, R. G., HILlYARD, S. A., \& LuCK, S. J. (1993) Electrocortical substrates of visual selective attention. In D. E. Meyer \& S. Kornblum (Eds.), Attention and performance XIV: Synergies in experimental psychology, artificial intelligence, and cognitive neuroscience (pp. 219-243). Cambridge, MA: MIT Press.

Maravita, A., Spence, C., Clarke, K., Husain, M., \& Driver, J. (2000). Vision and touch through the looking glass in a case of crossmodal extinction. NeuroReport, 11, 3521-3526.

Maravita, A., Spence, C., Sergent, C., \& Driver, J. (2002). Seeing your own touched hands in a mirror modulates cross-modal interactions. Psychological Science, 13, 350-356.

Meyer, V., Gross, C. G., \& Teuber, H. L. (1963). Effect of knowledge of site of stimulation on the threshold for pressure sensitivity. Perceptual \& Motor Skills, 16, 637-640.

MONDOR, T. A., \& ZATORRE, R. J. (1995). Shifting and focusing auditory spatial attention. Journal of Experimental Psychology: Human Perception \& Performance, 21, 387-409.

Moscovitch, M., \& BeHRMANN, M. (1994). Coding of spatial information in the somatosensory system: Evidence from patients with neglect following parietal lobe damage. Journal of Cognitive Neuroscience, 6, 151-155.

NIELSEN, T.I. (1963). Volition: A new experimental approach. Scandinavian Journal of Psychology, 4, 225-230.

PaVAni, F., SPEnCE, C., \& Driver, J. (2000). Visual capture of touch: Out-of-the-body experiences with rubber gloves. Psychological Science, 11, 353-359.

PENFIELD, W., \& RASMussen, T. (1950). The cerebral cortex of man. New York: Macmillan.

Ponzo, M. (1910). Intorno ad alcune illusioni nel campo delle sensazioni tattili, sull'illusione di Aristotele e fenomeni analoghi [On some tactile illusions, Aristotle's illusion, and similar phenomena]. Archiv für die Gesamte Psychologie, 16, 307-345.
Posner, M. I., \& CoHen, Y. (1984). Components of visual orienting. In H. Bouma \& D. G. Bouwhuis (Eds.), Attention and performance $X$ : Control of language processes (pp. 531-556). Hillsdale, NJ: Erlbaum.

RAMACHANDRAN, V. S., \& Rogers-RAMachandRan, D. (1996). Synaesthesia in phantom limbs induced with mirrors. Proceedings of the Royal Society of London: Series B, 263, 377-386.

RAmachandran, V. S., Rogers-RAmachandran, D., \& Cobb, S. (1995). Touching the phantom limb. Nature, 377, 489-490.

Rinker, M. A., \& Craig, J. C. (1994). The effect of spatial orientation on the perception of moving tactile stimuli. Perception \& Psychophysics, 56, 356-362.

RöDER, B., SPENCE, C., \& RöSLER, F. (2000). Inhibition of return and oculomotor control in the blind. NeuroReport, 11, 3043-3045.

Sathian, K., Greenspan, A. I., \& Wolf, S. L. (2000). Doing it with mirrors: A case study of a novel approach to neurohabilitation. $\mathrm{Neu}$ rohabilitation \& Neural Repair, 14, 73-76.

Shore, D. I., Spry, E., \& Spence, C. (2002). Confusing the mind by crossing the hands. Cognitive Brain Research, 14, 153-163.

SPENCE, C. (2001). Crossmodal attentional capture: A controversy resolved? In C. Folk \& B. Gibson (Eds.), Attention, distraction and action: Multiple perspectives on attentional capture (pp. 231-262). Amsterdam: Elsevier.

Spence, C., \& Driver, J. (1996). Audiovisual links in endogenous covert spatial attention. Journal of Experimental Psychology: Human Perception \& Performance, 22, 1005-1030.

SPENCE, C., \& DRIVER, J. (1997). Audiovisual links in exogenous covert spatial orienting. Perception \& Psychophysics, 59, 1-22.

Spence, C., \& Driver, J. (EDS.) (2004). Crossmodal space and crossmodal attention. Oxford: Oxford University Press.

Spence, C., Harris, C., \& Zampini, M. (2003, July). The "Japanese illusion" revisited? Impaired vibrotactile movement discrimination with interleaved fingers. Paper presented at the EPS Meeting, Reading, U.K.

Spence, C., Kingstone, A., Shore, D. I., \& Gazzaniga, M. S. (2001). Representation of visuotactile space in the split brain. Psychological Science, 12, 90-93

Spence, C., Lloyd, D., McGlone, F., Nicholls, M. E. R., \& DrIVER, J. (2000). Inhibition of return is supramodal: A demonstration between all possible pairings of vision, touch and audition. Experimental Brain Research, 134, 42-48.

SpEnCE, C., \& McGlone, F. P. (2001). Reflexive spatial orienting of tactile attention. Experimental Brain Research, 141, 324-330.

Spence, C., Pavani, F., \& Driver, J. (1998). What crossing the hands can reveal about crossmodal links in spatial attention. Abstracts of the Psychonomic Society, 3, 13.

Spence, C., Pavani, F., \& Driver, J. (2000). Crossmodal links between vision and touch in covert endogenous spatial attention. Journal of Experimental Psychology: Human Perception \& Performance, 26, 1298-1319.

Spence, C., Pavani, F., Maravita, A., \& Holmes, N. (2004). Multisensory contributions to the 3-D representation of visuotactile peripersonal space in humans: Evidence from the crossmodal congruency task. Journal of Physiology, 98, 171-189.

Spence, C., Shore, D. I., Gazzaniga, M. S., Soto-Faraco, S., \& KingStone, A. (2001). Failure to remap visuotactile space across the midline in the split-brain. Canadian Journal of Experimental Psychology, 55, 135-142.

Spence, C., Shore, D. I., \& Klein, R. M. (2001). Multisensory prior entry. Journal of Experimental Psychology: General, 130, 799-832.

Spence, C., \& Walton, M. (2004). Cross-modal congruency and visual capture in a visual elevation-discrimination task. Experimental Brain Research, 154, 113-120.

StYles, E. A. (1997). The psychology of attention. Hove, U.K.: Psychology Press.

Tastevin, J. (1937). En partant de l'expérience d'Aristote: Les déplacements artificiels des parties du corps ne sont pas suivis par le sentiment de ces parties ni par les sensations qu'on peut y produire [Starting from Aristotle's illusion: The artificial displacements of body parts are not followed by feeling in these parts or by the sensations which can be produced there]. L'Encephale, 1, 57-84, 140-158.

Tinazzi, M., Ferrari, G., Zampini, M., \& Aglioti, S. M. (2000). Neuropsychological evidence that somatic stimuli are spatially coded 
according to multiple frames of reference in a stroke patient with tactile extinction. Neuroscience Letters, 287, 133-136.

Treisman, A. [M.] (1998). Feature binding, attention and object perception. Philosophical Transactions of the Royal Society of London: Series $B$, 353, 1295-1306.

TrEISMAN, A. M., \& GeladE, G. (1980). A feature-integration theory of attention. Cognitive Psychology, 12, 97-136.

WeILAND, B. A. (1960). The interaction of space and time in cutaneous perception. American Journal of Psychology, 73, 248-255.

WeISENBERGER, J. M. (1994). Vibrotactile temporal masking: Effects of multiple maskers. Journal of the Acoustical Society of America, 95, 2213-2220.

WEISENBERGER, J. M., \& CRAIG, J. C. (1982). A tactile metacontrast effect. Perception \& Psychophysics, 31, 530-536.

Yamamoto, S., \& KitazaWa, S. (2001). Reversal of subjective temporal order due to arm crossing. Nature Neuroscience, $\underline{\mathbf{4}}$, 759-765.

\section{NOTES}

1. When placed flat on the table, the direction of motion of the two tactile arrays could be right to left or left to right across the thumb and finger pads. When the array on the thumb presented a right-to-left motion, the right-to-left motion on the finger was congruent in somatotopic, external, and egocentric coordinate systems. However, when the participant held the cube (opposing the finger and the thumb, with the tactile arrays perpendicular to the horizontal plane), the same right-toleft motion stimuli would now move in exactly the same directions as before in terms of the skin surface (i.e., in somatotopic coordinates), whereas within egocentrically and externally based coordinate frames, this same display would have a bottom-up direction on the thumb and a top-down direction across the finger (i.e., incongruent).

2. In order to avoid any concerns regarding the different number of blocks (the participants completed three blocks in Experiment 1 vs. four blocks in Experiment 2) and any potential confound in interpreting the interaction because of the overall difference in RTs across the two experiments, we conducted two additional data analyses. First, we repeated the same ANOVA as that reported in the main text, but now including just the first three blocks of trials from Experiment 2. The congruency effect was still significant $[F(1,14)=18.2, p<.001$, for RTs; $F(1,14)=22.9, p<.001$, for accuracy], RTs were again faster in Experiment 2 than in Experiment $1[F(1,14)=7.1, p<.05]$, and performance was also more accurate $[F(1,14)=6.7, p<.05]$. Critically, the interaction was still significant in terms of RTs $[F(1,14)=7.6, p<$ $.05]$ and accuracy $[F(1,14)=8.9, p<.01]$. In the second ANOVA, we repeated the main analysis, but now excluding the fastest 2 participants in Experiment 2 and the slowest 2 participants in Experiment 1. In this case, there was no difference in overall RTs between the two experiments $[F(1,10)=3.0, p=.115]$. However, the congruency effect was still significant overall $[F(1,10)=24.1, p<.001$, for RTs; $F(1,10)=$ $14.9, p<.005$, for accuracy], and more important for present purposes, the interaction between experiment and congruency was significant $[F(1,10)=6.9, p<.05$, for RTs; $F(1,10)=6.4, p<.05$, for accuracy $]$.

3 . But note that the participants may have been able to see the position of their hands momentarily during the feedback in the case of erroneous responses (since the four LEDs at the potential target and dis- tractor locations were illuminated briefly to give error feedback). This, together with the fact that (as in other studies) the participants could see where they placed their own hands when they were getting ready for the experiment (i.e., at the start of each block of trials), may imply that they could have built up and, possibly, maintained an internal visual representation of their body position. However, the potential information obtained through maintaining a visual image of the hands and arms (a confound that it is actually very difficult, if not impossible, to avoid completely) contrasts sharply with the continuous flow of visual information provided under normal viewing conditions and with the continuously available proprioceptive cues available throughout the experiments reported here.

4. This normally takes a few seconds. Although visual capture can take place for static limbs, the illusion is more powerful under conditions in which the hands are moved in synchrony (cf. Botvinick \& Cohen, 1998; Holmes, Crozier, \& Spence, 2004; Nielsen, 1963).

5. In an attempt to check whether previous experience with the mirror, by itself, had an influence on the results, we performed a new analysis on the error data separating the group of participants who started with the mirror condition and those who started without the mirror. As is reported in the main analyses, the congruency effect was larger in the visually close condition than in the far condition. However, the order of blocks factor did not reach significance or participate in any significant interaction with the other factors (all $F_{\mathrm{S}}<1$ ). It seems then, that previous knowledge of the mirror manipulation did not influence the data very much. This is perhaps not surprising, since we never attempted (nor was it really possible) to hide the nature of the mirror manipulation from the participants anyway.

6. The failure to find such an interference effect in Evans and Craig's (1991) previous study stands as an isolated example. There are several possible methodological reasons for this null result. On the basis of the research outlined here, the predictability of the target/distractor location appears to be one strong modulator of the magnitude of the congruency effect found and a potential reason for the particular pattern of results reported by Evans and colleagues in their study.

7. Many of these sensations are associated with the impression that the phantom limb is paralyzed in awkward and uncomfortable postures. In some cases, the mirror box has proved effective in releasing the phantom limb from this painful paralysis through the patient's seeing the reflected image of the preserved limb as it moves about (i.e., Ramachandran et al., 1995).

8. Note, however, that many of these effects have been obtained using temporal processing tasks, suggesting, perhaps, the existence of confusions about what happened when and where in touch (see Brigner, 1984; Jones, 1956; Kitazawa, 2002; Shore et al., 2002; Weiland, 1960). By contrast, the present results show clear evidence (through the effects of spatial distractors) that real or virtual changes in posture also influence purely spatial aspects of tactile perception.

(Manuscript received May 15, 2003; revision accepted for publication November 21, 2003.) 